\title{
A Marfan syndrome gene expression phenotype in cultured skin
} fibroblasts

\author{
Zizhen Yao1, Jochen C Jaeger ${ }^{1,7}$, Walter L Ruzzo ${ }^{1,2}$, Cecile Z Morale ${ }^{3,8}$, \\ Mary Emond ${ }^{4}$, Uta Francke ${ }^{5}$, Dianna M Milewicz ${ }^{6}$, Stephen M Schwartz ${ }^{3}$ and \\ Eileen R Mulvihill*3,9
}

\begin{abstract}
Address: ${ }^{1}$ Department of Computer Science and Engineering, University of Washington, Seattle, Washington 98195, USA, ${ }^{2}$ Department of Genome Sciences, University of Washington, Seattle, Washington 98195, USA, ${ }^{3}$ Department of Pathology, University of Washington, Seattle, Washington 98195, USA, ${ }^{4}$ Department of Biostatistics, University of Washington, Seattle Washington 98195, USA, ${ }^{5}$ Departments of Genetics and Pediatrics, Stanford University, Stanford, CA 94305-5323, USA, 6University of Texas Medical School at Houston, 6431 Fannin, MSB 1.614, Houston, TX 77030, USA, ${ }^{7}$ Hamilton Robotics, Via Crusch 8, Bonaduz, Switzerland, ${ }^{8}$ Trubion Pharmaceuticals Inc., Seattle, Washington 98121 , USA and ${ }^{9}$ PO Box 33, Villanueva, NM 87583, USA

Email: Zizhen Yao - yzizhen@cs.washington.edu; Jochen C Jaeger - jjaeger@hamilton.ch; Walter L Ruzzo - ruzzo@cs.washington.edu; Cecile Z Morale - cmorales@trubion.com; Mary Emond - emond@u.washington.edu; Uta Francke - ufrancke@stanford.edu; Dianna M Milewicz - dianna.m.milewicz@uth.tmc.edu; Stephen M Schwartz - steves@u.washington.edu;

Eileen R Mulvihill* - e.mulvihill@comcast.net

* Corresponding author
\end{abstract}

Published: 12 September 2007

BMC Genomics 2007, 8:319 doi:10.1/86//47/-2164-8-319
Received: 4 December 2006

Accepted: 12 September 2007

This article is available from: http://www.biomedcentral.com/|47|-2/64/8/3।9

(c) 2007 Yao et al; licensee BioMed Central Ltd.

This is an Open Access article distributed under the terms of the Creative Commons Attribution License (http://creativecommons.org/licenses/by/2.0), which permits unrestricted use, distribution, and reproduction in any medium, provided the original work is properly cited.

\begin{abstract}
Background: Marfan syndrome (MFS) is a heritable connective tissue disorder caused by mutations in the fibrillin-I gene. This syndrome constitutes a significant identifiable subtype of aortic aneurysmal disease, accounting for over $5 \%$ of ascending and thoracic aortic aneurysms.

Results: We used spotted membrane DNA macroarrays to identify genes whose altered expression levels may contribute to the phenotype of the disease. Our analysis of 4132 genes identified a subset with significant expression differences between skin fibroblast cultures from unaffected controls versus cultures from affected individuals with known fibrillin-I mutations. Subsequently, 10 genes were chosen for validation by quantitative RT-PCR.

Conclusion: Differential expression of many of the validated genes was associated with MFS samples when an additional group of unaffected and MFS affected subjects were analyzed ( $p$-value $<3 \times 10^{-6}$ under the null hypothesis that expression levels in cultured fibroblasts are unaffected by MFS status). An unexpected observation was the range of individual gene expression. In unaffected control subjects, expression ranges exceeding 10 fold were seen in many of the genes selected for qRT-PCR validation. The variation in expression in the MFS affected subjects was even greater.
\end{abstract}




\section{Background}

Aneurysm and dissection are major diseases of the aorta and are often asymptomatic until a life-threatening event like ischemic organ damage or rupture occurs. Marfan Syndrome (MFS) is a diverse yet clinically recognized subgroup of people at risk for aneurysm, including dissecting aneurysm, and constitutes a significant fraction (estimated at $5-7.5 \%[1,2])$ of all individuals with ascending and thoracic aortic aneurysmal disease. MFS incidence is estimated to be 1 in 5-10,000 [3]. Our long-term goal is to develop an assay that will identify people at risk for aneurysm before the disease process has reached an advanced state. This report is a small step in that direction.

In this study, we focus on individuals diagnosed with Marfan syndrome. The prevalence of MFS combined with its clinical recognition makes it an excellent model system for studies on aneurysmal disease. MFS is an autosomal dominant heritable disorder caused by mutations in the fibrillin-1 (FBN1) gene [4,5], with more than 500 unique mutations identified [6]. FBN1 mutations show a high degree of penetrance but considerable inter- and intrafamilial variability in their phenotype [3]. The variable penetrance suggests that environmental factors and/or disease modifying genes also contribute to the phenotype. Neonatal MFS correlates to mutations within exons 2432 and MFS defined by mutations in exons 59-65 carry a reduced risk of aortic pathology. Large-scale comparisons between MFS individuals with premature termination mutations and cysteine substitutions in FBN1 revealed significant differences in ocular, skeletal and hypermobility features but no difference in the frequency of ascending aortic aneurysm [7,8]. Apart from these observations, determining the nature of the mutation (a time and labor intensive process) does not improve prediction of the severity of the disease, the risk of aneurysm development or of its progression $[7,9]$. These limited genotype-phenotype correlations suggest that genes other than FBN1 may significantly influence the phenotype, and their identification may lead to a more informative test of risk.

Fibroblasts are not smooth muscle cells. However, in culture they display a stable phenotype with stress fibers composed of cytoplasmic actins and a splice variant of cellular fibronectin [10]. The increased mechanical stress on dermal fibroblasts seeded at low density produces a cell culture population consisting of 70-80\% myofibroblasts. The term "myofibroblast" was proposed over 30 years ago to describe the fibroblasts that appeared in granulation tissue at the sight of open wounds [11]. Recently, it has been recognized that Thy- 1 surface expression defines a subpopulation of fibroblasts capable of differentiating into myofibroblasts [12]. We can detect Thy-1 expression in both affected and unaffected skin fibroblasts by array and have confirmed that observation by quantitative real time polymerase chain reaction (qRT-PCR, data not shown). Thus, the skin cultures we used were "myofibroblast" like.

In the last several years, use of DNA microarrays to analyze gene expression has emerged as a promising technology for disease classification and prognosis and for identification of genes that could be potential causes, biomarkers or drug targets $[13,14,14-18]$. However, there are limits to the sensitivity of microarrays for detecting genes expressed at low levels as well as additional confounding problems associated with arrays [19-23]. Consequently, in common with most recent studies [20,24], we go beyond mere classification by independently validating expression levels using quantitative qRT-PCR and validating the results in a second population.

In the present study, we used total RNA in oligo dT primed cDNA reactions to identify an expression phenotype associated with the MFS genotype in cultured skin fibroblasts. Our results show a clearly recognizable expression phenotype in cultured fibroblasts. We of course do not expect exactly the same expression phenotype in aortic smooth muscle cells, but we do expect some overlap in the perturbed pathways, as they share the same root cause. Some of the identified genes, including elastin and several collagens, are obvious targets for roles in the development and maintenance of the extracellular matrix environment and cell-matrix contacts. Additional genes validated by qRTPCR analysis, including the vitamin D receptor, programmed cell death-10 and the LIM domain only 7, may represent genes that will provide new insight into the disease process. Our ability to detect a MFS expression phenotype in cultured fibroblasts provides both a simple method for large-scale screening and a basis for mechanistic studies of the genes identified as differentially expressed.

\section{Results}

An overview of our experimental design is shown in Figure 1 . In brief, we analyzed total RNA from 36 subjects by spotted membrane DNA macroarray. We used Research Genetics spotted cDNA membrane arrays to analyze gene expression in fibroblast cultures, 17 with characterized mutations in the FBN1 gene ( 7 missense, all cysteine substitutions, 9 nonsense and 1 multi-exon deletion; 14 of 17 had an aortic phenotype) and 19 unaffected controls (details in Table 1). We selected differentially expressed genes between MFS and unaffected samples based on estimated False Discovery Rate (FDR)[25]. We calculated the 4132 p-values for the two-sample t-tests associated with the expression differences between affected and control for each gene on the array. At a q-value (estimated FDR) threshold of $0.001,283$ genes (265 with identifiable unique Unigene ID numbers) out of a total of 4132 are 


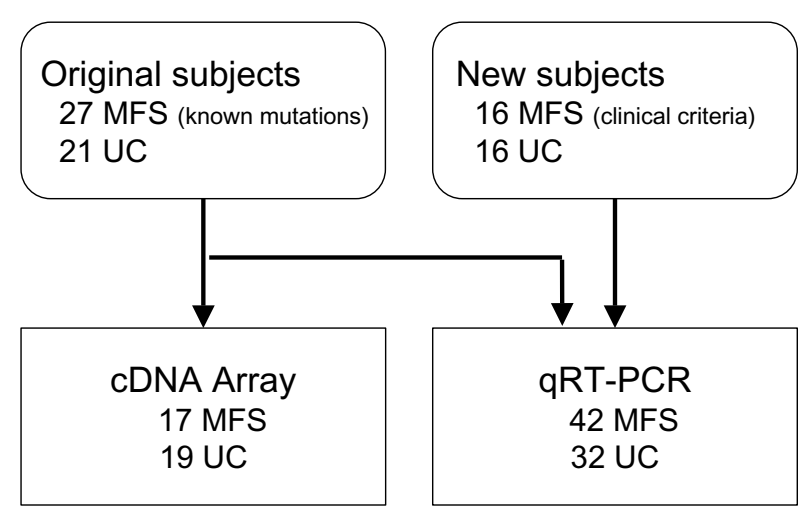

\section{Figure I}

Overview of the experimental design. We used spotted membrane DNA arrays to characterize gene expression from MFS samples with known FBNI mutations and from unaffected control samples. Following analysis of both groups, we selected a set of genes for validation by qRT-PCR, using a majority of the original samples. A new population of I 6 probable MFS, all with an aortic phenotype, and I 6 UC samples were used to test independently whether the selected genes were differentially expressed between the two groups. See Table I for details about subjects included in each experiment.

selected as differentially expressed in MFS vs. unaffected. The top ranking genes based on ratio are listed in Table 2 [see Additional File 1 for the entire list]. At the same qvalue threshold, we found 175 differentially expressed genes (165 with unique Unigene ID) between missense and unaffected samples, and 111 differentially expressed genes (106 with unique Unigene ID) between nonsense and unaffected samples. The q-values are only approximate, since their validity depends on various assumptions such as normality of the expression levels within groups. To globally assess the reliability of these test results while making fewer statistical assumptions, we performed a permutation test (see Methods) to determine if the large number of significant genes we found could easily have arisen by chance. Of 50,000 random permutations for MFS and unaffected samples, no permutations have more than 283 differentially expressed genes, making the array results highly statistically significant, with an empirical pvalue smaller than 0.00002 . Furthermore, the only permutations exhibiting a substantial number of significant genes were those permutations that happened to separate most of the MFS subjects from most of the unaffected controls. Over forty five thousand permutations showed no significant genes, and the average number called significant was only 0.45 , making it very likely that our estimated false discovery rate of 0.001 is conservative. [See Additional File 1 for the complete 4132-gene dataset.]

\section{Quantitative RT-PCR}

We performed duplicate quantitative RT-PCR assays on total RNA from 74 subjects (Table 1 ) to measure the concentration of 12 mRNAs [see Additional file 2]. We chose 10 genes based on the degree of ratio change, small qvalue and the availability of Applied Biosystems predetermined assay reagents (PDARs). We included two additional genes, GUSB and TBP as internal references. Behaviors of these genes in the array are summarized in Table 3. The qRT-PCR data for a given subject are generally positively correlated with the array results for the same 10 genes from the same RNA sample, with a mean Spearman (rank) correlation of 0.46 (0.35 among unaffected subjects, 0.52 among MFS subjects). Figure 2 summarizes qRT-PCR results for all 74 subjects, and Table 4 additionally summarizes qRT-PCR results for the array and new subjects separately.

It is noteworthy that the expression values in the qRT-PCR assays do not appear to be normally distributed. E.g., boxand-whisker plots of normally distributed data analogous to Figure 2 would be expected to show less than $1 \%$ of the samples as "outliers" (the triangles plotted outside the "whisker" ranges, which encompass \pm 2.7 times the estimated standard deviation), whereas Figure 2 has 3.7 times as many outliers among the UC samples and 9.4 times as many among MFS samples (also see Table 5). In response to this apparent non-normality, we used the non-parametric Wilcoxon rank sum test to evaluate the significance of gene expression differences between groups, a more conservative choice than the usual t-test in such a circumstance. Under the null hypothesis that data for the two groups are sampled from the same (unspecified) continuous distribution, the test is sensitive to a shift in the location (e.g. mean and median) of the distributions (but not necessarily sensitive to a shift in variance, as seen in most genes here).

Table 4 shows that when all of the samples are combined, 6 of 10 genes were validated. Under the null hypothesis that the genes are independent and not influenced by MFS status, the chance that the qRT-PCR results would confirm 6 of 10 predictions as being statistically significant (Wilcoxon p value $<0.05$ ) is less than $2.8 \times 10^{-6}$. Fold changes and Wilcoxon p values for all 10 genes are shown in Table 4 based on all 74 subjects and separately, on 30 of the original 36 array subjects and 32 new subjects (Table 1). Among 10 genes selected based on the array experiments, 8 show consistent direction of difference by qRT-PCR on the same array subjects, and 5 of these 8 genes are statistically significant. Similarly, 6 of these 10 genes show the same direction, and are significant, when tested on new subjects, as are 6 of 10 when tested on all subjects. Four of the 10 genes are significant in all three sets of subjects 
Table I: FBNI mutations [8, 60, $61-3]$

\begin{tabular}{|c|c|c|c|c|c|c|c|c|c|c|c|c|c|}
\hline & \multicolumn{7}{|c|}{ MFS Affected (known mutations) } & \multicolumn{6}{|c|}{ Unaffected Controls (UC) } \\
\hline \multirow[b]{2}{*}{1} & \multirow{2}{*}{$\begin{array}{l}\text { Subject } \\
\text { FB } 969\end{array}$} & \multirow{2}{*}{$\frac{\text { Sex }}{F}$} & \multirow{2}{*}{$\begin{array}{r}\text { Age } \\
53\end{array}$} & \multirow{2}{*}{$\begin{array}{l}\text { Mutation } \\
\text { C628X }\end{array}$} & \multicolumn{2}{|c|}{ Platform } & \multirow{2}{*}{$\begin{array}{c}\text { Ref. } \\
30\end{array}$} & \multicolumn{2}{|c|}{ Subject } & \multirow{2}{*}{$\frac{\text { Sex }}{F}$} & \multirow{2}{*}{$\begin{array}{r}\text { Age } \\
74\end{array}$} & \multicolumn{2}{|c|}{ Platform } \\
\hline & & & & & $A$ & $\mathrm{~T}$ & & UC & I & & & & $\mathrm{T}$ \\
\hline 2 & FB 992 & M & 49 & R86IX & A & $\mathrm{T}$ & 30 & UC & 2 & $\mathrm{~F}$ & 34 & & $\mathrm{~T}$ \\
\hline 3 & GD I05। & $\mathrm{F}$ & 45 & PTC ex 24 & $A$ & $\mathrm{~T}$ & DMUP & UC & 3 & $\mathrm{~F}$ & 34 & $A$ & $\mathrm{~T}$ \\
\hline 4 & FB I 234 & $\mathrm{~F}$ & 34 & NII57X & A & $\mathrm{T}$ & 30 & UC & 4 & $\mathrm{~F}$ & 34 & $A$ & $\mathrm{~T}$ \\
\hline 5 & FB 773 & $\mathrm{~F}$ & 48 & DII9IX & $A$ & $T$ & 30,61 & UC & 5 & $\mathrm{~F}$ & 38 & $A$ & $\mathrm{~T}$ \\
\hline 6 & FB 857 & $M$ & 16 & RII92X & & $\mathrm{T}$ & 30 & UC & 6 & $\mathrm{~F}$ & 35 & $A$ & $\mathrm{~T}$ \\
\hline 7 & FB 75I & $\mathrm{F}$ & 54 & LI4I2X & & $\mathrm{T}$ & 30 & UC & 7 & $\mathrm{~F}$ & 15 & $A$ & $\mathrm{~T}$ \\
\hline 8 & FB 997 & $\mathrm{~F}$ & 48 & RI523X & & $\mathrm{T}$ & 30 & UC & 8 & $\mathrm{~F}$ & II & $A$ & \\
\hline 9 & FB I 286 & $\mathrm{~F}$ & 33 & R2057X & A & $\mathrm{T}$ & 30 & UC & 9 & $\mathrm{~F}$ & 5 & $A$ & \\
\hline 10 & FR 60 & $M$ & 7 & PTC ex 63 & $A$ & $\mathrm{~T}$ & DMUP & UC & 10 & $F$ & 25 & $A$ & $\mathrm{~T}$ \\
\hline II & GD 021 & $M$ & 17 & PTC ex 63 & A & $\mathrm{T}$ & DMUP & UC & 11 & $\mathrm{~F}$ & 49 & $A$ & \\
\hline 12 & GD 032 & $\mathrm{~F}$ & 9 & PTC ex 64 & $A$ & & DMUP & UC & 12 & $\mathrm{~F}$ & 40 & $A$ & \\
\hline 13 & FB 836 & $\mathrm{~F}$ & 31 & C832Y & $A$ & $\mathrm{~T}$ & 8,61 & UC & 13 & $M$ & 72 & $A$ & $\mathrm{~T}$ \\
\hline 14 & FB 837 & $M$ & 10 & $\mathrm{C} 832 \mathrm{Y}$ & A & $\mathrm{T}$ & 8,61 & UC & 14 & $M$ & 49 & A & $\mathrm{T}$ \\
\hline 15 & FB 783 & $\mathrm{~F}$ & 33 & CIIITY & $A$ & $\mathrm{~T}$ & 61 & UC & 15 & $M$ & 36 & $A$ & $\mathrm{~T}$ \\
\hline 16 & FB 984 & $M$ & 22 & CIITIR & & $\mathrm{T}$ & 8 & UC & 16 & $M$ & 43 & $A$ & $\mathrm{~T}$ \\
\hline 17 & FB 1069 & $\mathrm{~F}$ & 4 & $\mathrm{Cl} 326 \mathrm{R}$ & & $\mathrm{T}$ & 8 & UC & 17 & $M$ & 35 & $A$ & $\mathrm{~T}$ \\
\hline 18 & FB 1040 & $\mathrm{~F}$ & 29 & CI36IY & $A$ & $\mathrm{~T}$ & 8 & UC & 18 & $M$ & 15 & $A$ & $\mathrm{~T}$ \\
\hline 19 & FB 882 & $\mathrm{~F}$ & 27 & $\mathrm{Cl} 402 \mathrm{~W}$ & & $\mathrm{~T}$ & 8 & UC & 19 & $M$ & 71 & $A$ & $\mathrm{~T}$ \\
\hline 20 & FB 88I & $M$ & 24 & CI589F & $A$ & $\mathrm{~T}$ & 61 & UC & 20 & $M$ & 14 & $A$ & $\mathrm{~T}$ \\
\hline 21 & FB 1627 & $M$ & 34 & C2038Y & $A$ & $\mathrm{~T}$ & UFUP & UC & 21 & $M$ & 50 & $A$ & \\
\hline 22 & FB 829 & $M$ & 21 & $\mathrm{C} 2053 \mathrm{~F}$ & & $\mathrm{~T}$ & 62 & & & & & & \\
\hline 23 & FB 1211 & $M$ & 20 & C2IIIR & $A$ & $\mathrm{~T}$ & UFUP & & & & & & \\
\hline 24 & FB 1359 & $M$ & 28 & $\mathrm{C} 2686 \mathrm{~F}$ & & $\mathrm{~T}$ & 62 & & & & & & \\
\hline 25 & FB 890 & $\mathrm{~F}$ & $4 \mathrm{mo}$ & del ex 44-46 & $A$ & $\mathrm{~T}$ & 63 & & & & & & \\
\hline 26 & FB 774 & $\mathrm{~F}$ & 40 & del ex $42-43$ & & $\mathrm{~T}$ & 63 & & & & & & \\
\hline 27 & FB 970 & $M$ & 38 & del ex 54 & & $\mathrm{~T}$ & 63 & & & & & & \\
\hline
\end{tabular}

Average Age 29

Average Age 37

Additional MFS Affected (unknown mutations)

Additional Unaffected Controls (UC)

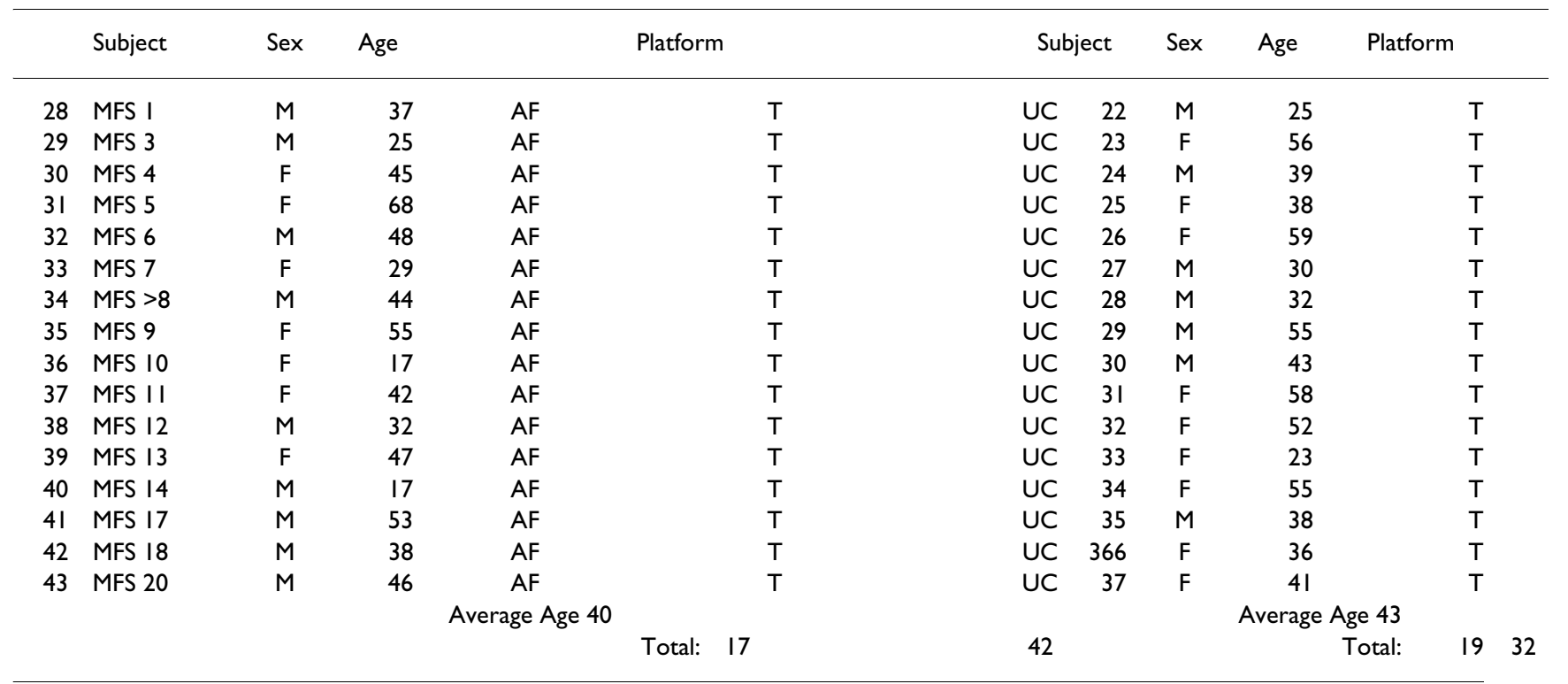


Table 2: Top 15 genes identified by ratio from the array dataset

\begin{tabular}{|c|c|c|c|c|c|c|}
\hline UG cluster ID & SwissProt Accession & Gene name & MFS NI & UC NI & Ratio MFS/UC & q-value \\
\hline Hs. 146688 & PTGES:OI4684 & Prostaglandin E synthase & 185 & 108 & I.7I & I.36E-03 \\
\hline Hs.90303 & TSC2:P498I5 & Tuberous sclerosis 2 & 60 & 37 & 1.64 & I.26E-04 \\
\hline Hs. 1872 & PCKI:P35558 & Phosphoenolpyruvate carboxykinase I & 249 & 157 & 1.59 & 4.IIE-03 \\
\hline Hs.310545 & SYTI:P2I 579 & Synaptotagmin I & 109 & 69 & 1.57 & $2.60 \mathrm{E}-03$ \\
\hline Hs. 144700 & EFNBI:P98I72 & Ephrin-BI & 296 & 191 & 1.56 & $4.84 \mathrm{E}-03$ \\
\hline Hs.334534 & GNS:PI5586 & Glucosamine ( $\mathrm{N}$-acetyl)-6-sulfatase & 173 & 112 & 1.54 & $5.23 \mathrm{E}-05$ \\
\hline Hs.386283 & ADAMI2:043I84 & Meltrin L & 44 & 73 & $1.65^{-1}$ & $5.08 \mathrm{E}-09$ \\
\hline Hs.5I6646 & CREBI:PI6220 & CAMP responsive element $\mathrm{BP}$ I & 193 & 339 & $1.76^{-1}$ & I.96E-05 \\
\hline Hs.489|42 & COLIA2:P08I23 & Collagen, type I, alpha 2 & 3663 & 6582 & $1.80^{-1}$ & $4.48 \mathrm{E}-06$ \\
\hline Hs. I 46447 & FBNI:P35555 & Fibrillin I & 124 & 233 & $1.89-1$ & $2.52 \mathrm{E}-10$ \\
\hline Hs.25058I & SMARCD2:Q92925 & SWI/SNF D2 & 1532 & 2963 & $1.93^{-1}$ & 5.99E-06 \\
\hline Hs.432862 & MARCH-VI:O60337 & RING-CH protein VI & 2091 & 4245 & $2.03^{-1}$ & 4.97E-09 \\
\hline Hs.55967 & SHOX2:060902 & Short stature homeobox 2 & 58 & 137 & $2.38^{-1}$ & $1.13 E-12$ \\
\hline Hs.443625 & COL3AI:P0246I & Collagen, type III, alpha I & 638 & 1538 & $2.4 I^{-1}$ & $2.06 \mathrm{E}-04$ \\
\hline Hs.2524l8 & ELN:PI5502 & Elastin & 35 & 117 & $3.34^{-1}$ & 2.19E-08 \\
\hline
\end{tabular}

Unigene ID identifies significant, differentially expressed genes based on expression ratio criteria. The top 6 down regulated and 9 up regulated genes are included. The normalized intensity $(\mathrm{NI})$ from the phosphorimager scan is listed to give a sense of the expression range. The $q$ values are computed based on $t$ test $p$ values. In this and subsequent tables, we show all ratios less than I as reciprocals. The entire $4 I 32$ gene list has been deposited into GEO (the Gene Expression Omnibus), accession number GSE8759.

showing that the array experiments were effective in detecting MFS associated genes.

The two genes marked N/A show marginally statistically significant changes in Array Subjects but very highly significant changes in the opposite direction in New Subjects. One of these genes is Fibrillin-1, the key gene implicated in MFS. In our array experiments, FBN1 was significantly repressed ( 1.89 fold, $\mathrm{q}=2.52 \times 10^{-10}$; Table 4) and qRT-PCR confirmed that its (geometric) mean level was 1.47 fold lower in MFS based on (predominantly) the same subjects ( $\mathrm{p}=0.052$, Table 5 "Array Subjects"). However, qRT-PCR shows that in "New Subjects" this gene is significantly more highly expressed in MFS than controls $\left(2.71\right.$ fold higher, $\left.\mathrm{p}=3.3 \times 10^{-9}\right)$. Viewed another way, over all subjects, the difference in mean FBN1 levels between affected and unaffected subjects is not statistically significant, but 22 of 42 MFS subjects have values more extreme than the most extreme value in the unaffected samples (9 lower, 13 higher). It seems likely that MFS subjects exhibit considerable heterogeneity in FBN1 mRNA levels and our original and new subject populations may not be equivalent samples. As was previously shown, nonsense mutations lead to nonsensemediated decay of the mutant mRNA and reduced fibrillin protein synthesis [26-28]. On the other hand, all the known FBN1 missense mutations used in this study are cysteine substitutions in calcium-binding EGF domains. FBN1 transcript levels and fibrillin protein synthesis were normal in these cells $[8,26,27]$. As the distribution of mutations types in the second set of clinically identified MFS subjects is unknown, they may not replicate the distribution of the two mutation classes in the original MFS subjects. An additional factor is a difference in cell culture

Table 3: Data summary of genes selected for validation by qRT-PCR

\begin{tabular}{|c|c|c|c|c|c|c|}
\hline SwissProt Accession & Gene name & PDAR & MFS NI & UC NI & Ratio MFS/UC & q value \\
\hline VDR:PI|473 & vitamin $\mathrm{D}$ receptor & Hs00I72II3_ml & 59 & 84 & $1.42^{-1}$ & 3.94E-I I \\
\hline FBNI:P35555 & fibrillin I & Hs00I7I79I_ml & 124 & 233 & $1.89-1$ & 2.52E- 10 \\
\hline INHBA:P08476 & inhibin, beta $A$ & Hs00I70l03_ml & 72 & 117 & $\left.1.6\right|^{-1}$ & $3.48 \mathrm{E}-10$ \\
\hline ELN:PI5502 & elastin & Hs00355783_ml & 35 & 117 & $3.34-1$ & 2.19E-08 \\
\hline COLIA2:P08I 23 & collagen, type I, alpha 2 & Hs00164099_m I & 3663 & 6582 & $1.80^{-1}$ & 4.48E-06 \\
\hline PCOLCE:QI5II3 & procollagen C-endopeptidase & Hs00I70I79_ml & 134 & 168 & $1.26^{-1}$ & 2.99E-04 \\
\hline PLOD2:000469 & lysine hydroxylase 2 & Hs00I68688_ml & 92 & 75 & 1.22 & 3.00E-04 \\
\hline PDCD / 0Q9BUL8 & programmed cell death 10 & Hs00200578_ml & 534 & 464 & 1.15 & I.29E-03 \\
\hline PTGES:OI4684 & prostaglandin E synthase & Hs006I0420_ml & 185 & 108 & 1.72 & I.36E-03 \\
\hline LM07:Q8WWII & LIM domain only 7 & Hs00245600_ml & 50 & 45 & 1.13 & 5.07E-03 \\
\hline
\end{tabular}

We selected 10 genes for validation based on their performance on the array. Column headings are as in Table 2, except PDAR: Applied Biosystems predetermined assay reagent identifier. 


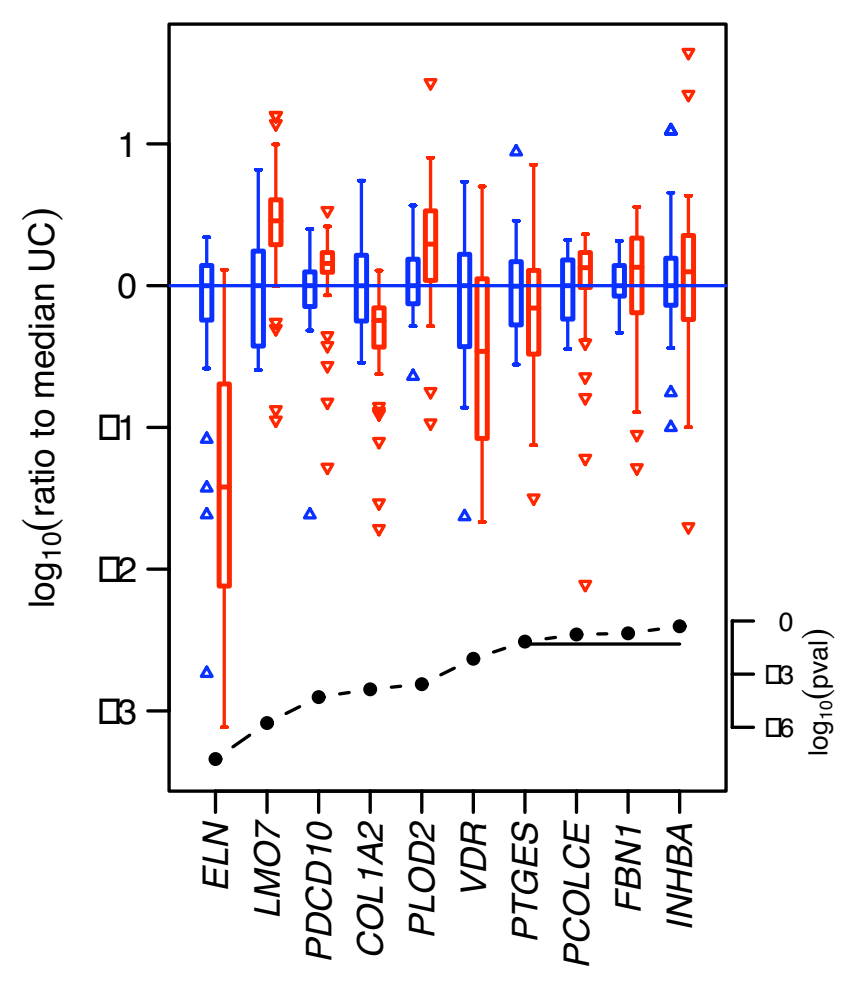

Figure 2

Validation by quantitative qRT-PCR. The figure presents a summary of the 10 genes selected for validation by qRT-PCR. Above each gene name are two "box-andwhisker" plots of expression levels for that gene across 32 unaffected control (UC) samples (left plot of each pair, blue, up-triangles) and 42 MFS affected samples (right, red, downtriangles). Vertical axis is $\log _{10}$ ratio of expression level to the median UC level. Each "box" shows the inter-quartile range (IQR), i.e., the range between the $25^{\text {th }}$ and $75^{\text {th }}$ percentiles of the log ratios; the horizontal line in each box is the 50th percentile (median). (Median log ratio for UC is always zero, by definition.) "Whiskers" (vertical lines) extend from each box to the most extreme values within I.5 times the IQR from the box; in normally distributed data this would on average encompass $99 \%$ of the values. Triangles mark more extreme points. The lower curve shows $\log _{10}$ ( $p$-value) for a Wilcoxon rank sum test of the null hypothesis that the UC and MFS distribution are identical; horizontal line marks the $p=0.05$ significance level. 6 of 10 genes have $p$-values $<0.05$ by this test. Most genes exhibit noticeably greater variability across the MFS samples than across UC samples, although the Wilcoxon test is not sensitive to this. To highlight one example, for Elastin (ELN), the middle $50 \%$ of the UC sample log ratios fall between -0.23 and +0.14 (i.e., the $25^{\text {th }}$ and $75^{\text {th }}$ percentiles of the values fall I.70-fold below and I.38-fold above the median, respectively), and all but 4 fall between -0.58 and +0.34 (4-fold below and 2.2-fold above median). In contrast, median Elastin level is 26 fold lower in MFS samples, only four MFS samples are above the UC median, and the null hypothesis has a p-value of $1.6 \times 10^{-8}$. passage number. Nearly all the UC and all new MFS subjects were used at passage 2 while the original MFS cultures were used between passage 3 and 6 . The second gene marked N/A in Table 4 is procollagen C-endopeptidase $(P C O L C E)$, another gene with a clear mechanistic role in maintenance of the extracellular matrix [28]. It is plausibly co-regulated with $F B N 1$, showing a moderately strong correlation to FBN1 in our qRT-PCR data. Given these uncertainties as to the homogeneity of our subject population with respect to these two genes, we considered that overall ratios and p-values were potentially misleading; hence we chose to omit them.

A surprising result of the qRT-PCR measurements, evident in Figure 2 and analyzed in more detail in Table 5, are the variability of expression of these genes, both in unaffected and especially in MFS subjects. We looked at both the full range of the data and at the interquartile range (IQR; the range between the $25^{\text {th }}$ and $75^{\text {th }}$ percentiles, quantifying the spread in the central majority of the population, a statistic that is very robust to the influence of outliers). In general, most genes in UC samples show interquartile ranges of at most 3-fold, with two showing roughly 4-fold changes (LMO7, VDR). IQR for MFS samples is similar, with a slight tendency to higher variability (e.g., higher mean and quartiles, and 6 of 10 showing greater variability across MFS samples than UC) and a few dramatic exceptions (VDR 11 fold; ELN 24 fold). Variability is more pronounced over the full range. In the UC samples the majority vary by 16-230 fold, and one by more than 1000 fold. MFS subjects show greater variability than UC samples in at least 8 of the 10 genes. The least variable gene (PDCD10) changes by more than 64-fold across MFS subjects, the majority vary by more than 225 -fold, with some (ELN and INHBA) showing subject-to-subject differences exceeding 1600-fold. Controlling for age, sex, or missense/nonsense mutations did not reduce the marked variability. (The effect of cell passage number (see Methods) could not be tested.)

For the long term, this variability emphasizes the importance of studies involving carefully targeted and/or large subject populations, and of developing simple assays for relevant phenotypic predictors, since small samples may be non-representative. For the purposes of the present study, variability complicates interpretation of the results for similar reasons. For example, in our previous discussion of FBN1 we remarked that it did not meet our criterion for a statistically significant shift in expression level in the "All Subjects" grouping, although it remains likely that FBN1 level is influenced by MFS status and specific types of FBN1 mutations. Similar remarks apply to other genes, where increased variability across MFS subjects suggests these genes are involved in disease processes, but not by a mechanism that consistently elevates or consistently 
Table 4: QRT-PCR validation and phenotype prediction

\begin{tabular}{|c|c|c|c|c|c|c|c|}
\hline \multirow{3}{*}{$\begin{array}{l}\text { Gene symbol } \\
V D R\end{array}$} & \multirow{3}{*}{$\begin{array}{c}\text { Array ratio } \\
1.42^{-1}\end{array}$} & \multicolumn{6}{|c|}{ qRT-PCR results: MFS/UC ratio (Wilcoxon p-value) } \\
\hline & & \multicolumn{2}{|c|}{ Array subjects } & \multicolumn{2}{|c|}{ New subjects } & \multicolumn{2}{|c|}{ All subjects } \\
\hline & & 4.59-1 & $(9.5 e-5)$ & 1.25 & $(8.1 \mathrm{e}-1)$ & $2.53-1$ & $(7.3 e-3)$ \\
\hline FBNI & $1.89-1$ & I.47-I & $(5.2 \mathrm{e}-2)$ & 2.71 & $(3.3 e-9)$ & $\mathrm{N} / \mathrm{A}$ & \\
\hline INHBA & $1.61^{-1}$ & 2.20 & $(I .1 \mathrm{e}-\mathrm{I})$ & $1.06-1$ & $(6.7 e-1)$ & 1.05 & $(5.0 \mathrm{e}-\mathrm{I})$ \\
\hline ELN & $3.34^{-1}$ & $12.53^{-1}$ & $(2.4 e-3)$ & $9.68^{-1}$ & $(2.3 e-4)$ & $15.04^{-1}$ & $(1.6 \mathrm{e}-8)$ \\
\hline COLIA2 & $1.80^{-1}$ & $1.24^{-1}$ & $(I . \mid \mathrm{e}-\mathrm{I})$ & $2.4 I-1$ & $(2.3 e-8)$ & $2.27-1$ & $(1.4 \mathrm{e}-4)$ \\
\hline PCOLCE & $1.26^{-1}$ & $1.27^{-1}$ & $(5.8 \mathrm{e}-2)$ & 2.02 & $(4.6 \mathrm{e}-6)$ & $\mathrm{N} / \mathrm{A}$ & \\
\hline PLOD2 & 1.22 & 3.01 & $(3.8 \mathrm{e}-4)$ & 1.69 & $(8.2 \mathrm{e}-3)$ & 1.85 & $(2.7 e-4)$ \\
\hline PDCD $/ 0$ & 1.15 & 1.98 & $(1.9 e-6)$ & 1.65 & $(4.8 e-3)$ & I.4I & $(5.0 \mathrm{e}-5)$ \\
\hline PTGES & 1.72 & $3.42^{-1}$ & $(5.8 e-4)$ & 1.88 & $(9.3 e-3)$ & $1.68^{-1}$ & $(6.8 e-2)$ \\
\hline LMO7 & 1.13 & 2.01 & (1.0e-3) & 4.67 & $(3.0 \mathrm{e}-6)$ & 2.77 & (1.7e-6) \\
\hline \multirow{2}{*}{\multicolumn{2}{|c|}{ Overall Significance }} & \multicolumn{2}{|c|}{ Array subjects } & \multicolumn{2}{|c|}{ New subjects } & \multicolumn{2}{|c|}{ All subjects } \\
\hline & & 5 & $(6.4 e-5)$ & 6 & $(2.8 e-6)$ & 6 & $(2.8 e-6)$ \\
\hline
\end{tabular}

Column 2: average MFS/UC ratio from array experiments. Remaining columns: ratios of geometric mean transcript abundances of MFS affected vs unaffected control for three subject groups, and Wilcoxon $p$ values for the null hypothesis of no between group differences. The three groups are: (I) "Array Subjects": 30 of the original 36 array subjects (I6 known FBNI mutations and I4 unaffected controls); (2) "New Subjects": 32 new subjects including 16 MFS based on clinical criteria and 16 unaffected controls; and (3) "All Subjects": 42 MFS affected compared to 32 unaffected (all of the above groups plus 10 additional characterized FBNI mutations and 2 additional controls). (See Table I for subject details.) Bold: array validation or significant $p$ value $(p<0.05)$. Italic: significant $p$-value but difference opposite to the expected direction. N/A: see text. The bottom section of the table provides a summative assessment: the (binomial) $p$-value of the observed number of significant $q R T-P C R$ validations $(p<0.05)$ under the null hypothesis of no between-group differences.

represses expression. These large variations in expression may limit the extent of our interpretations and will require more focused studies and/or additional statistical power before an association with the MFS phenotype can be established firmly. Alternatively the expression distribution might be influenced by other factors such as the genotypes of FBN1 or disease modifying genes that we have not yet identified.

\section{Discussion}

We conducted a small-scale gene expression analysis in MFS affected subjects. We hypothesized that subsets of the

Table 5: Gene variability by qRT-PCR

\begin{tabular}{|c|c|c|c|c|c|c|c|c|}
\hline \multirow[b]{2}{*}{ Gene } & \multicolumn{3}{|c|}{ Interquartile range } & \multicolumn{3}{|c|}{ Full range } & \multicolumn{2}{|c|}{ \# Outliers } \\
\hline & UC & MFS & MFS/UC & UC & MFS & MFS/UC & UC & MFS \\
\hline COLIA2 & 2.9 & 1.9 & 0.7 & 19.2 & 66.1 & 3.4 & 0 & 6 \\
\hline ELN & 2.4 & 24.3 & 10.4 & 1190.4 & 1687.3 & 1.4 & 4 & 0 \\
\hline FBNI & 1.6 & 3.3 & 2.0 & 4.4 & 69.3 & 15.6 & 0 & 2 \\
\hline INHBA & 2.2 & 3.7 & 1.7 & 124.5 & 2218.2 & 17.8 & 4 & 3 \\
\hline LMO7 & 4.7 & 2.0 & 0.4 & 25.9 & $|4| . \mid$ & 5.4 & 0 & 7 \\
\hline PCOLCE & 2.6 & 1.8 & 0.7 & 5.9 & 295.5 & 50.3 & 0 & 5 \\
\hline PDCD $/ 0$ & 1.8 & 1.4 & 0.8 & 103.6 & 64.7 & 0.6 & 1 & 6 \\
\hline PLOD2 & 2.0 & 3.1 & 1.5 & 16.0 & 251.2 & 15.7 & 1 & 3 \\
\hline PTGES & 2.8 & 3.7 & 1.3 & 31.7 & 225.0 & 7.1 & I & I \\
\hline VDR & 3.9 & 11.3 & 2.9 & 230.3 & 233.0 & 1.0 & I & 0 \\
\hline $\min$ & 1.6 & 1.4 & 0.4 & 4.4 & 64.7 & 0.6 & 0.0 & 0.0 \\
\hline median & 2.5 & 3.2 & 1.4 & 28.8 & 229.0 & 6.3 & 1.0 & 3.0 \\
\hline mean & 2.7 & 5.7 & 2.2 & 175.2 & 525.1 & 11.8 & 1.2 & 3.3 \\
\hline $\max$ & 4.7 & 24.3 & 10.4 & 1190.4 & 2218.2 & 50.3 & 4.0 & 7.0 \\
\hline$\#>1$ & & & 6 & & & 8 & & \\
\hline
\end{tabular}

For the 10 genes validated by qRT-PCR, we summarize variability across $32 \mathrm{UC}$ and 42 MFS samples. Interquartile Range: fold change between the $25^{\text {th }}$ and $75^{\text {th }}$ percentiles. Full Range: fold change between the most extreme pair of samples. \#Outliers: number of outliers (triangles in Figure 2). If the data were normally distributed, approximately I\% of the points would so identified, i.e., on average 0.32 per gene for UC and 0.42 per gene for MFS samples. 
expression phenotype might indicate genetic and epigenetic factors that are causal or predictive of the MFS diseased state, and more generally, of the aneurysmal state. To conduct this test, we hypothesized that primary cultures of skin fibroblasts derived from punch biopsies could be a simple, robust model. An important advantage to using this system is the ability to collect samples from large numbers of both affected and unaffected subjects. Fibroblasts express FBN1, and distinct fibrillin protein phenotypes have been identified in fibroblast cultures from MFS individuals $[26,27,29]$. The protein profiles have been linked to distinct classes of FBN1 mutations and clinical phenotypes $[8,30]$.

We used spotted membrane DNA array screening to identify gene expression changes associated with FBN1 mutations. The most important outcome of this study is the identification of a small number of differentially expressed genes that distinguished skin fibroblasts of MFS affected individuals from the fibroblasts of unaffected controls. Our DNA array experiments identified a subset of genes that were validated in a new group of subjects. Due to the size of our study we chose to focus on the expression pattern of affected vs. unaffected and chose 10 genes for validation.

We can speculate on how a number of the validated genes could contribute to the MFS phenotype in a mechanistic way. MFS is a heritable fibrillinopathy where defects in the synthesis and/or assembly of fibrillin-1 microfibrils lead to impairment in the elastic fibrils that confer resilience and recoil in elastic tissues [31,32]. For over a decade it was thought that the microfibrillar component of elastic fibers provided a three-dimensional scaffold for the assembly of elastin in the process of elastogenesis [33]. The histopathological abnormalities of aneurysmal vessels include abnormal extracellular matrix protein accumulation, fragmentation and disorganization of the elastic fibers in the medial layer of the vascular wall, and a generalized loss of elastin content [31,32]. Analysis of mouse models of fibrillin- 1 deficiency suggested that the primary defect is not in elastogenesis but rather in elastic fiber homeostasis [34]. Characterization of the mouse model led to a model of acquired elastolysis [35] with evidence suggesting that the altered elastic fiber structure could change the expression phenotype of the underlying vascular smooth muscle cells, resulting in the increased expression of several proteases. A number of investigators have identified the association of specific types of FBN1 mutations with an increased susceptibility to protease digestion [9] and with the ability of FBN1 fragments to up regulate protease expression in culture [36]. In contrast, our study identifies elastin as one of the most significantly regulated genes in the affected cell lines. Surprisingly, elastin mRNA is markedly decreased (Table $4 ; 15.0^{-1}, \mathrm{p}=1.6$ $\times 10^{-8}$ ) suggesting a very early defect in elastogenesis that starts at the level of elastin transcription or the regulation of elastin message stability. Interestingly, the vitamin D receptor was also significantly decreased in MFS subjects (Table $4 ; 2.5^{-1}, \mathrm{p}=7.3 \times 10^{-3}$ ). Vitamin $\mathrm{D}$ is known to decrease elastin mRNA by decreasing message stability in cultured cells [37]. Vitamin D treatment also is known to decrease both elastin content and the number of elastic lamellae in the aortas of animals treated pre and postnatally

[38].

Recent publications have also described an increasing role for transforming growth factor beta (TGF-beta) in the expression of the MFS phenotype. Mutations in both TGFbeta receptors have been identified in humans with heritable forms of aneurysm [39-41]. In a mouse model of MFS the mutant phenotype includes developmental abnormalities of the distal alveolae of the lung, associated with increased TGF-beta protein and activity $[42,43]$. Our arrays did not detect any significant difference in TGF beta expression level, while a related family member, INHBA, was not validated by the qRT-PCR analysis. More recently, TGF-beta antagonists, including a neutralizing antibody to TGF-beta and losartan (an angiotensin II type receptor ATI blocker) were able to prevent and reverse aneurysmal progression in the same mouse model [44]. We found significant differences in the expression of a number of genes in this pathway including VDR (Table $4 ; 2.53^{-1}, \mathrm{p}=7.3 \times$ $10^{-3}$ ) and TSC2 (Table 2; 1.64, $\mathrm{q}=1.3 \times 10^{-4}$ ). The vitamin $\mathrm{D}$ receptor $(V D R)$ is a negative regulator of TGF-beta transcriptional activation [45] and as mentioned above is significantly decreased in MFS skin fibroblasts. In contrast, the tuberous sclerosis complex 2 (TSC2), a potent activator of TGF-beta transcription, is expressed more highly in MFS fibroblasts (Table 2; 1.64, q = $1.3 \times 10^{-4}$ ). Another gene, LMO7 (a protein that connects the nectin-afadin and E-cadherin-catenin systems through alpha-actinin and therefore regulates cell adhesion) [46] is induced by TGF-beta and is significantly elevated in MFS fibroblasts (Table $4 ; 2.77, \mathrm{p}=1.7 \times 10^{-6}$ ). The behavior of all three genes is consistent with enhanced TGF-beta activity that could contribute to the aneurysmal phenotype if smooth muscle cell loss or differentiated phenotype contributes to the pathological process.

Our data also identify several matrix metalloproteases, including ADAM12 (Table 2; $1.65^{-1}, \mathrm{q}=5.1 \times 10^{-9}$ ), MMP1 $\left(1.14, \mathrm{q}=2.1 \times 10^{-2}\right)$ and the metalloproteinase inhibitor TIMP3 $\left(1.39^{-1}, \mathrm{q}=6.0 \times 10^{-6}\right)$, with significant differences in expression between the unaffected and affected group. Several collagens including $C O L 3 A 1$ (Table 2; 2.41-1, $\mathrm{q}=2.1 \times 10^{-4}$ ), COL1A2 (Table 4; 2.27-1, $\mathrm{p}=1.4 \times 10^{-4}$ ) and the lysine hydroxylase PLOD2 (Table $4 ; 1.85, \mathrm{p}=2.7 \times 10^{-4}$ ) (which forms hydroxylysine residues in collagen which serve as attachment sites for carbo- 
hydrates that contribute to the stability of the intermolecular collagen cross-links) are also significantly changed. The appearance of these genes suggests a broad change in the extracellular fibrillar structure and composition in affected cells. Many of these matrix proteins and modifying enzymes are regulated by TGF-beta. $[47,44,48]$.

PDCD10 (programmed cell death gene 10 ) is increased in the MFS samples (Table 4; 1.41, $\mathrm{p}=5.0 \times 10^{-5}$ ). PDCD10 was recently identified as one of three genes responsible for a heritable form of cerebral cavernous malformations (CCM3) characterized by abnormally enlarged capillary cavities causing seizures and hemorrhages [49]. These vascular malformations appear to result from a failure in vascular morphogenesis and/or remodeling. In CCM, the endothelial tubes continue to expand and stabilizing pericytes are not recruited. The result is a vessel with an enormously dilated lumen and increased fragility [50].

When we initiated these studies we had two important concerns. First, a priori, it is not obvious that cultured skin fibroblasts would exhibit significant expression differences for other genes in MFS subjects. However, the skin is one of the connective tissues affected in MFS, and our experiments confirm a significant MFS expression phenotype in cultured fibroblasts. The second concern is that these differences may not be relevant to aneurysmal disease, believed to be largely a disease of smooth muscle cells. Obviously, we cannot be sure that the gene changes discovered in fibroblasts are the same gene changes found in aortic smooth muscle cells, but, given that the two cell types share commonalities in their morphology, extracellular matrix environment and the common fibrillin defect, it is reasonable to suspect that they also share some relevant core changes in fibrillin-related pathways. While our fibroblast study cannot answer this question, it did identify some differentially expressed genes whose known roles are suggestive of involvement in SMC tissue failure. A third concern arose during the course of the study namely, the large sample-to-sample variation in gene expression levels. This variability needs to be recognized as an important confounding factor in expression analysis studies. It also supports our decision to use skin fibroblasts rather than aortic SMC as our sample source.

\section{Conclusion}

Our ultimate goal is to use genome-wide expression analysis to identify and classify people at risk for developing aneurysms of the ascending thoracic aorta. Our preliminary data support the idea that there are common mechanisms triggered by mutations in FBN1 that lead to identifiable differences in gene expression between unaffected control and MFS affected cultured fibroblasts. Some of these changes appear to be downstream from TGF-beta activation. While the limited phenotype described here is only a partial description of the potential "mutation associated" expression profile, the results suggest that a complete genomic screen of several hundred MFS vs. unaffected cell lines, using FBN1 mutation detection, microarray expression analysis and qRT-PCR validation of possible biomarkers, could lead to the identification of genes that contribute to the mechanistic events that initiate vessel wall destruction.

\section{Methods}

\section{Cell culture, RNA isolation, and array hybridization}

Study participants were recruited under an institutional review board approved protocol and informed consent. A skin punch biopsy was used to establish a fibroblast cell culture. Unaffected control subjects were selected from patients visiting dermatology clinics. All of the cell strains (in the array group, between passage 2 and 6 and in the New Subject group, passage 2) were grown to confluence in SmGM2 + 10\% FBS + bullet kit containing recombinant EGF, FGF and insulin (BioWhittaker). After reaching confluence, the medium was modified to include ascorbic acid at $50-\mu \mathrm{g} / \mathrm{ml}$ [51]. Fresh medium was added after 24 hours. At $48 \mathrm{~h}$, total RNA was isolated using a guanidinium iso-thiocyanate-phenol-chloroform extraction protocol [52]. Each sample was analyzed for quality and quantity by UV spectroscopy and gel electrophoresis. For hybridization to individual arrays, $1 \mu \mathrm{g}$ of total RNA was used to synthesize ${ }^{33} \mathrm{P}$-dCTP labeled first strand cDNA with Invitrogen Superscript II. Generally, each sample was labeled twice and hybridized to duplicate Research Genetics GF211 arrays (4 arrays in all). Each array was hybridized with 30-60 million cpm of probe for $18 \mathrm{~h}$ then washed extensively at $50^{\circ} \mathrm{C}$ in $0.5 \times \mathrm{SSC}+0.1 \%$ SDS. Multiple exposures were collected on a Storm phosphorimager. Images were imported using Research Genetics Pathways 3 software, and processed as described in "Array Data Normalization."

\section{Quantitative RT-PCR}

Validation of the array results was done using Applied Biosystems pre-designed qRT-PCR primer and probe sets (PDAR's, see ABI P/N 4333458_a.pdf for the complete protocol). These assays use a standard PCR format where all the assays are performed with the same PCR cycling conditions. Quantification is based on calculating a ratio to an included calibrator sample. Our calibrator was 50\% Stratagene Universal Target total RNA and 50\% total RNA pooled from the 36 array subjects listed in Table 1. We synthesized first strand cDNA using random primers and the ABI High Capacity cDNA Archive Kit (P/N 4322171). Duplicate PCR reactions were set up in a final volume of 25 ul using 10 ng equivalents of input total RNA. We used the ABI 7900 machine with the standard cycling protocol, increasing the number of cycles to 40. GUSB and TBP were 
used as an internal reference in all of the samples and calibrator. The delta delta Ct method [54] was used to calculate ratios of each gene in each sample against the calibrator RNA. Both references gave similar results and we present the GUSB quantification.

\section{Array data normalization}

Normalization refers to the process of attempting to correct for the many sources of systematic variation in cDNA array. In outline, we do the following. The median of $\log _{10}$-transformed expression values for each gene across all exposures of all arrays is calculated, and serves as a common baseline for comparison between experiments. Then, for each exposure, we compute a smooth "local" (loess) regression line between its log intensities and the median levels. The resulting regression function transforms the expression values of each exposure to the scale of the common baseline while capturing nonlinear effects. Finally, we combine data from multiple exposures of an array by taking their medians. This technique builds on earlier work $[54,55]$ and more details are reported in [56]. One innovation introduced here is the following: to reduce the impact of outliers and differentially expressed genes on the inferred levels of other genes, the loess regression is based on only a "stable" subset of genes uniformly chosen across all intensity levels. Specifically, for each gene we calculate the rank of its measured intensity in each exposure, and the median absolute deviation (MAD) of its rank across exposures. We sort all $n$ genes by increasing median intensity and partition them into $\mathrm{n} / 5$ groups of consecutive genes. From each group of 5 , the gene with the minimum MAD statistic is chosen. This selection of $\mathrm{n} / 5$ genes comprises the stable subset of genes on which our regression is based. Other approaches to normalization based on stable genes have been proposed $[57,58]$ but those techniques seem more complex, and choose stable genes in ways that potentially yield sparse coverage of some regions of the intensity spectrum, consequently increasing the influence of anomalous or differentially expressed genes on the normalization in those regions. As intensity-dependent non-linearity is a key concern in our normalization, uniform representation of intensities in the stable subset is valuable.

Correlation analyses and ANOVA tests identified possible technical biases in a subset of hybridizations. As a consequence, hybridization data from seven Marfan and two unaffected samples were eliminated from further study (indicated by blanks in the platform columns in Table 1). Most of these subjects were included in the qRT-PCR analysis and appear in the "All Subjects" category of Table 4.

\section{Differential expression and permutation analysis}

We used standard t-test p-values to rank genes for evidence of differential expression, coupled with FDR analy- sis, and selected a conservative $\mathrm{q}=.001$ cutoff threshold. We chose the classical t-test for its familiarity and simplicity. The non-parametric Wilcoxon rank sum test yielded very similar gene rankings. We decided against the commonly used SAM-statistic [59] because of an interest in genes with low expression levels. SAM is biased somewhat against such genes [60].)

To avoid dependence on parametric assumptions about the array data, we performed a permutation test to determine if the large number of significant genes we found could easily have arisen by chance. For a comparison of subject groups $\mathrm{A}$ and $\mathrm{B}$, we combined $\mathrm{A}$ and $\mathrm{B}$ and then randomly split the combined group into two groups $\mathrm{A}^{\prime}$ and $B^{\prime}$, such that groups $A$ and $A^{\prime}$ are the same size, groups $B$ and $B^{\prime}$ are the same size, and the technical replicates of any one sample are either all in $\mathrm{A}^{\prime}$ or all in $\mathrm{B}^{\prime}$. Hence, the disease status labels were randomly permuted to determine whether the true disease was responsible for the observed result of 283 genes with $\mathrm{q}<0.001$. We then computed the number of differentially expressed genes at the same cutoff threshold (FDR $<0.001$ ) in each such random partition.

\section{qRT-PCR data analysis}

As part of the statistical analysis of the qRT-PCR data, we performed per-gene regression tests for age, sex and/or group effects on expression levels (in addition to MFS status). There were no significant sex effects in any of the tests. Age effects were marginally significant for two genes, but not after Bonferroni correction, and furthermore would not have altered the significance of MFS status even if included. For some genes, inclusion of subject in "new" vs. "original" subject groups (Table 1) was significant, but again correction for this effect did not alter the significance of MFS status. Hence, we have chosen to present results only for the simplest model, which does not attempt to adjust for any of these covariates.

Significance of differential expression was quantified by Wilcoxon rank sum test; see Figure 2, Table 4. "Overall significance" results reported in the last line of Table 4 follow from a simple binomial model.

\section{Abbreviations}

MFS- Marfan Syndrome.

MS- Missense mutation.

NS- Nonsense mutation.

UC- Unaffected Control.

qRT-PCR- Quantitative Real Time Polymerase Chain Reaction. 
FDR- False Discovery Rate.

SAM- Statistical Analysis of Microarrays.

IQR- Interquartile Range.

MAD- median absolute deviation.

PDAR- Pre-designed Assay Reagents.

PTC - Premature termination codon.

Del Ex - Deleted exon(s).

\section{Authors' contributions}

EM conceived of and designed the study. CZM and EM performed all of the experiments. ZY, JCJ, WLR and ME performed all the statistical analysis, UF and DM contributed the Marfan skin fibroblasts with known mutations, and DM contributed most of the normal skin fibroblasts. SMS contributed to the design of the study. WLR, ERM and SMS are co-senior authors. All of the authors contributed to the writing of the manuscript.

\section{Additional material}

\section{Additional file 1}

Statistical analysis of the normalized array data. The data provided represent the statistical analysis of the normalized data from the array subjects identified (A) in Table 1.

Click here for file

[http://www.biomedcentral.com/content/supplementary/14712164-8-319-S1.xls]

\section{Additional file 2}

Delta delta CT values for the qRT-PCR analysis. The data provide the average delta delta CT values for all the subjects identified (T) in Table 1. Click here for file

[http://www.biomedcentral.com/content/supplementary/1471-

2164-8-319-S2.xls]

\section{Acknowledgements}

CZM and EM were supported by AHA grant $0151515 Z$; JCJ and WLR by NSF DBI -9974498; ZY, WLR, EM, CZM, ME and SMS by NIH POI HL72262 and WLR by NIH-NIEHS P30ES07033. We would like to thank Dr. Alexander Clowes and members of the Marfan community whose contributions purchased most of the qRT-PCR primers, probes and reagents, Research Genetics for donating spotted membrane DNA arrays and the National Marfan Foundation who arranged to have skin biopsies taken from volunteers at their annual clinic conference events in Baltimore and Chicago.

\section{References}

I. Coady MA, Rizzo JA, Goldstein LJ, Elefteriades JA: Natural history, pathogenesis, and etiology of thoracic aortic aneurysms and dissections. Cardiol Clin 1999, I 7:615-635.
2. Januzzi JL, Marayati F, Mehta RH, Cooper JV, O'Gara PT, Sechtem U, Bossone E, Evangelista A, Oh JK, Nienaber CA, Eagle KA, Isselbacher EM: Comparison of aortic dissection in patients with and without Marfan's syndrome (results from the International Registry of Aortic Dissection). Am J Cardiol 2004, 94:400-402.

3. Pyeritz RE: The Marfan syndrome. Annu Rev Med 2000, $5 \mathrm{I}: 48 \mathrm{I}-5 \mathrm{IO}$

4. Dietz HC, Cutting GR, Pyeritz RE, Maslen CL, Sakai LY, Corson GM, Puffenberger EG, Hamosh A, Nanthakumar EJ, Curristin SM, .: Marfan syndrome caused by a recurrent de novo missense mutation in the fibrillin gene. Nature 1991, 352:337-339.

5. Dietz HC, Pyeritz RE, Hall BD, Cadle RG, Hamosh A, Schwartz J, Meyers DA, Francomano CA: The Marfan syndrome locus: confirmation of assignment to chromosome 15 and identification of tightly linked markers at I5qI5-q2I.3. Genomics I99I, 9:355-36I.

6. Collod-Beroud G, Le Bourdelles S, Ades L, Ala-Kokko L, Booms P, Boxer M, Child A, Comeglio P, de Paepe A, Hyland JC, Holman K, Kaitila I, Loeys B, Matyas G, Nuytinck L, Peltonen L, Rantamaki T, Robinson P, Steinmann B, Junien C, Beroud C, Boileau C: Update of the UMD-FBNI mutation database and creation of an FBNI polymorphism database. Hum Mutat 2003, 22:199-208.

7. Loeys B, De Backer J, Van Acker P, Wettinck K, Pals G, Nuytinck L, Coucke $P$, de Paepe $A$ : Comprehensive molecular screening of the FBNI gene favors locus homogeneity of classical Marfan syndrome. Hum Mutat 2004, 24: I40-I 46.

8. Schrijver I, Liu W, Brenn T, Furthmayr H, Francke U: Cysteine Substitutions in Epidermal Growth Factor-Like Domains of Fibrillin-I: Distinct Effects on Biochemical and Clinical Phenotypes. Am J Hum Genet 1999, 65: 1007-1020.

9. Robinson PN, Booms P, Katzke S, Ladewig M, Neumann L, Palz M, Pregla R, Tiecke F, Rosenberg T: Mutations of FBNI and genotype-phenotype correlations in Marfan syndrome and related fibrillinopathies. Hum Mutat 2002, 20:153-161.

10. Gabbiani G: The myofibroblast in wound healing and fibrocontractive diseases. J Pathol 2003, 200:500-503.

II. Desmouliere A, Chaponnier C, Gabbiani G: Tissue repair, contraction, and the myofibroblast. Wound Repair Regen 2005, 13:7-12.

12. Koumas L, Smith TJ, Feldon S, Blumberg N, Phipps RP: Thy-I expression in human fibroblast subsets defines myofibroblastic or lipofibroblastic phenotypes. Am J Pathol 2003, 163:1291-1300.

13. Mariadason JM, Arango D, Augenlicht LH: Customizing chemotherapy for colon cancer: the potential of gene expression profiling. Drug Resist Updat 2004, 7:209-218.

14. Ramaswamy S, Tamayo P, Rifkin R, Mukherjee S, Yeang $\mathrm{CH}$, Angelo M, Ladd C, Reich M, Latulippe E, Mesirov JP, Poggio T, Gerald W, Loda M, Lander ES, Golub TR: Multiclass cancer diagnosis using tumor gene expression signatures. Proc Natl Acad Sci U S A 200I, 98:15I49-15I54.

15. van de Vijver MJ, He YD, van't Veer LJ, Dai H, Hart AA, Voskuil DW, Schreiber G], Peterse JL, Roberts C, Marton MJ, Parrish M, Atsma D, Witteveen A, Glas A, Delahaye L, van V, Bartelink H, Rodenhuis S, Rutgers ET, Friend SH, Bernards R: A gene-expression signature as a predictor of survival in breast cancer. N Engl J Med 2002, 347:1999-2009.

16. Kote-Jarai Z, Williams RD, Cattini N, Copeland M, Giddings I, Wooster R, tePoele RH, Workman P, Gusterson B, Peacock J, Gui G, Campbell $C$, Eeles R: Gene expression profiling after radiationinduced DNA damage is strongly predictive of BRCAI mutation carrier status. Clin Cancer Res 2004, 1 0:958-963.

17. Khan J, Wei JS, Ringner M, Saal LH, Ladanyi M, Westermann F, Berthold F, Schwab M, Antonescu CR, Peterson C, Meltzer PS: Classification and diagnostic prediction of cancers using gene expression profiling and artificial neural networks. Nat Med 200 I, 7:673-679.

18. Lee YF, John M, Falconer A, Edwards S, Clark J, Flohr P, Roe T, Wang R, Shipley J, Grimer RJ, Mangham DC, Thomas JM, Fisher C, Judson I, Cooper CS: A gene expression signature associated with metastatic outcome in human leiomyosarcomas. Cancer Res 2004, 64:7201-7204.

19. Ebert BL, Golub TR: Genomic approaches to hematologic malignancies. Blood 2004, 104:923-932.

20. Tan PK, Downey TJ, Spitznagel EL Jr., Xu P, Fu D, Dimitrov DS, Lempicki RA, Raaka BM, Cam MC: Evaluation of gene expression 
measurements from commercial microarray platforms. Nucleic Acids Res 2003, 31:5676-5684.

21. Lee JK, Bussey KJ, Gwadry FG, Reinhold W, Riddick G, Pelletier SL, Nishizuka S, Szakacs G, Annereau JP, Shankavaram U, Lababidi S, Smith LH, Gottesman MM, Weinstein JN: Comparing cDNA and oligonucleotide array data: concordance of gene expression across platforms for the NCl-60 cancer cells. Genome Biol 2003, 4:R82.

22. Jarvinen AK, Hautaniemi S, Edgren $\mathrm{H}$, Auvinen $\mathrm{P}$, Saarela J, Kallioniemi OP, Monni O: Are data from different gene expression microarray platforms comparable? Genomics 2004, 83: I I64-II68.

23. Kuo WP, Kim EY, Trimarchi J, Jenssen TK, Vinterbo SA, Ohno-Machado $L$ : $A$ primer on gene expression and microarrays for machine learning researchers. J Biomed Inform 2004, 37:293-303.

24. Bullinger L, Dohner K, Bair E, Frohling S, Schlenk RF, Tibshirani R, Dohner H, Pollack JR: Use of gene-expression profiling to identify prognostic subclasses in adult acute myeloid leukemia. $\mathrm{N}$ Engl J Med 2004, 350:1605-1616.

25. Storey JD: A direct approach to false discovery rates. Jorunal of the Royal Statistical Society 2002, Series B, 64:479-498.

26. Aoyama T, Francke U, Dietz HC, Furthmayr H: Quantitative differences in biosynthesis and extracellular deposition of fibrillin in cultured fibroblasts distinguish five groups of Marfan syndrome patients and suggest distinct pathogenetic mechanisms. J Clin Invest 1994, 94:130-137.

27. Brenn T, Aoyama T, Francke U, Furthmayr H: Dermal fibroblast culture as a model system for studies of fibrillin assembly and pathogenetic mechanisms: defects in distinct groups of individuals with Marfan's syndrome. Lab Invest 1996, 75:389-402.

28. Prockop DJ, Sieron AL, Li SW: Procollagen N-proteinase and procollagen $\mathrm{C}$-proteinase. Two unusual metalloproteinases that are essential for procollagen processing probably have important roles in development and cell signaling. Matrix Biol 1998, 16:399-408.

29. Milewicz DM, Pyeritz RE, Crawford ES, Byers PH: Marfan syndrome: defective synthesis, secretion, and extracellular matrix formation of fibrillin by cultured dermal fibroblasts. Clin Invest 1992, 89:79-86.

30. Schrijver I, Liu W, Odom R, Brenn T, Oefner P, Furthmayr H, Francke $\mathrm{U}$ : Premature termination mutations in FBNI: distinct effects on differential allelic expression and on protein and clinical phenotypes. Am J Hum Genet 2002, 7 I:223-237.

31. Milewicz DM, Urban Z, Boyd C: Genetic disorders of the elastic fiber system. Matrix Biol 2000, 19:47I-480.

32. Urban Z, Boyd CD: Elastic-fiber pathologies: primary defects in assembly-and secondary disorders in transport and delivery. Am J Hum Genet 2000, 67:4-7.

33. Robert L: Aging of the vascular wall and atherogenesis: role of the elastin- laminin receptor [published erratum appears in Atherosclerosis 1996 Sep 27; 126(1):173]. Atherosclerosis 1996, 123:169-179.

34. Pereira L, Lee SY, Gayraud B, Andrikopoulos K, Shapiro SD, Bunton T, Giery NJ, Dietz HC, Sakai LY, Ramirez F: Pathogenetic sequence for aneurysm revealed in mice underexpressing fibrillin-I. Proc Natl Acad Sci U S A 1999, 96:381 9-3823.

35. Bunton TE, Biery NJ, Myers L, Gayraud B, Ramirez F, Dietz HC: Phenotypic alteration of vascular smooth muscle cells precedes elastolysis in a mouse model of Marfan syndrome. Circ Res 200I, 88:37-43.

36. Booms P, Pregla R, Ney A, Barthel F, Reinhardt DP, Pletschacher A, Mundlos $S$, Robinson PN: RGD-containing fibrillin-I fragments upregulate matrix metalloproteinase expression in cell culture: a potential factor in the pathogenesis of the Marfan syndrome. Hum Genet 2005, I 16:51-6I.

37. Pierce RA, Kolodziej ME, Parks WC: I,25-Dihydroxyvitamin D3 represses tropoelastin expression by a posttranscriptional mechanism. J Biol Chem 1992, 267: I I593-1 I599.

38. Norman P, Moss I, Sian M, Gosling M, Powell J: Maternal and postnatal vitamin $D$ ingestion influences rat aortic structure, function and elastin content. Cardiovasc Res 2002, 55:369-374.

39. Loeys BL, Chen J, Neptune ER, Judge DP, Podowski M, Holm T, Meyers J, Leitch CC, Katsanis N, Sharifi N, Xu FL, Myers LA, Spevak PJ, Cameron DE, De Backer J, Hellemans J, Chen Y, Davis EC, Webb CL, Kress W, Coucke P, Rifkin DB, De Paepe AM, Dietz HC: A syndrome of altered cardiovascular, craniofacial, neurocogni- tive and skeletal development caused by mutations in TGFBR I or TGFBR2. Nat Genet 2005, 37:275-28I.

40. Loeys BL, Schwarze U, Holm T, Callewaert BL, Thomas GH, Pannu $\mathrm{H}$, De Backer JF, Oswald GL, Symoens S, Manouvrier S, Roberts AE, Faravelli F, Greco MA, Pyeritz RE, Milewicz DM, Coucke PJ, Cameron DE, Braverman AC, Byers PH, De Paepe AM, Dietz HC: Aneurysm syndromes caused by mutations in the TGF-beta receptor. N Engl J Med 2006, 355:788-798.

4I. Mizuguchi T, Collod-Beroud G, Akiyama T, Abifadel M, Harada N, Morisaki T, Allard D, Varret M, Claustres M, Morisaki H, Ihara M, Kinoshita A, Yoshiura K, Junien C, Kajii T, Jondeau G, Ohta T, Kishino T, Furukawa Y, Nakamura Y, Niikawa N, Boileau C, Matsumoto N: Heterozygous TGFBR2 mutations in Marfan syndrome. Nat Genet 2004, 36:855-860.

42. Neptune ER, Frischmeyer PA, Arking DE, Myers L, Bunton TE, Gayraud B, Ramirez F, Sakai LY, Dietz HC: Dysregulation of TGF. beta activation contributes to pathogenesis in Marfan syndrome. Nat Genet 2003, 33:407-4II.

43. Ng CM, Cheng A, Myers LA, Martinez-Murillo F, Jie C, Bedja D, Gabrielson KL, Hausladen JM, Mecham RP, Judge DP, Dietz HC: TGFbeta-dependent pathogenesis of mitral valve prolapse in a mouse model of Marfan syndrome. J Clin Invest 2004, I | 4: I586-I592.

44. Habashi JP, Judge DP, Holm TM, Cohn RD, Loeys BL, Cooper TK, Myers L, Klein EC, Liu G, Calvi C, Podowski M, Neptune ER, Halushka MK, Bedja D, Gabrielson K, Rifkin DB, Carta L, Ramirez F, Huso DL, Dietz HC: Losartan, an ATI antagonist, prevents aortic aneurysm in a mouse model of Marfan syndrome. Science 2006, 3I2:II7-I2I.

45. Wu Y, Craig TA, Lutz WH, Kumar R: Identification of I alpha,25dihydroxyvitamin $D 3$ response elements in the human transforming growth factor beta 2 gene. Biochemistry 1999, 38:2654-2660.

46. Ooshio T, Irie K, Morimoto K, Fukuhara A, Imai T, Takai Y. Involvement of LMO7 in the association of two cell-cell adhesion molecules, nectin and E-cadherin, through afadin and alphaactinin in epithelial cells. J Biol Chem 2004, 279:3।365-3|373.

47. Le Pabic H, L'Helgoualc'h A, Coutant A, Wewer UM, Baffet G, Clement $B$, Theret $N$ : Involvement of the serine/threonine p70S6 kinase in TGF-betal-induced ADAMI2 expression in cultured human hepatic stellate cells. J Hepatol 2005, 43: 1038-1044.

48. Kwak HJ, Park MJ, Cho H, Park CM, Moon SI, Lee HC, Park IC, Kim $\mathrm{MS}$, Rhee $\mathrm{CH}$, Hong SI: Transforming growth factor-betal induces tissue inhibitor of metalloproteinase-I expression via activation of extracellular signal-regulated kinase and SpI in human fibrosarcoma cells. Mol Cancer Res 2006, 4:209-220.

49. Bergametti F, Denier C, Labauge P, Arnoult M, Boetto S, Clanet M, Coubes P, Echenne B, Ibrahim R, Irthum B, Jacquet G, Lonjon M, Moreau JJ, Neau JP, Parker F, Tremoulet M, Tournier-Lasserve E: Mutations within the programmed cell death 10 gene cause cerebral cavernous malformations. Am J Hum Genet 2005, 76:42-5I

50. Coucouvanis E, Martin GR: Signals for death and survival: a twostep mechanism for cavitation in the vertebrate embryo. Cell 1995, 83:279-287.

51. Jain MK, Layne MD, Watanabe M, Chin MT, Feinberg MW, Sibinga NE, Hsieh CM, Yet SF, Stemple DL, Lee ME: In vitro system for differentiating pluripotent neural crest cells into smooth muscle cells. J Biol Chem 1998, 273:5993-5996.

52. Chomczynski P, Sacchi N: Single-step method of RNA isolation by acid guanidinium thiocyanate-phenol-chloroform extraction. Anal Biochem 1987, 162:156-159.

53. Lossos IS, Czerwinski DK, Alizadeh AA, Wechser MA, Tibshirani R, Botstein D, Levy R: Prediction of survival in diffuse large-B-cell lymphoma based on the expression of six genes. $N$ Engl J Med 2004, 350: |828-1837.

54. Dozmorov I, Galecki A, Chang Y, Krzesicki R, Vergara M, Miller RA: Gene expression profile of long-lived snell dwarf mice. J Gerontol A Biol Sci Med Sci 2002, 57:B99-108.

55. Yang YH, Dudoit S, Luu P, Lin DM, Peng V, Ngai J, Speed TP: Normalization for CDNA microarray data: a robust composite method addressing single and multiple slide systematic variation. Nucleic Acids Res 2002, 30:el5. 
56. Mulvihill ER, Jaeger J, Sengupta R, Ruzzo WL, Reimer C, Lukito S, Schwartz SM: Atherosclerotic plaque smooth muscle cells have a distinct phenotype. Arterioscler Thromb Vasc Biol 2004, 24: I283-I289.

57. Kepler TB, Crosby L, Morgan KT: Normalization and analysis of DNA microarray data by self-consistency and local regression. Genome Biol 2002, 3:RESEARCH0037.

58. Schadt EE, Li C, Ellis B, Wong WH: Feature extraction and normalization algorithms for high-density oligonucleotide gene expression array data. J Cell Biochem Suppl 200I, Suppl 37:120-125.

59. Tusher VG, Tibshirani R, Chu G: Significance analysis of microarrays applied to the ionizing radiation response. Proc Natl Acad Sci U S A 200I, 98:5116-5I21.

60. Storey JD, Tibshirani R: Statistical methods for identifying differentially expressed genes in DNA microarrays. Methods Mol Biol 2003, 224: 149-157.

61. Tynan K, Comeau K, Pearson M, Wilgenbus P, Levitt D, Gasner C, et al.: Mutation screening of complete fibrillin-I coding sequence: report of five new mutations, including two in 8cysteine domains. Hum Mol Genet 1993, 2:1813-1821.

62. Liu W, Schrijver I, Brenn T, Furthmayr H, Francke U: Multi-exon deletions of the FBNI gene in Marfan syndrome. BMC Med Genet 200I, 2:II.

63. Liu WO, Oefner PJ, Qian C, Odom RS, Francke U: Denaturing HPLC-identified novel FBNI mutations, polymorphisms, and sequence variants in Marfan syndrome and related connective tissue disorders. Genet Test 1997, I(4):237-42.

Publish with Bio Med Central and every scientist can read your work free of charge

"BioMed Central will be the most significant development for disseminating the results of biomedical research in our lifetime. "

Sir Paul Nurse, Cancer Research UK

Your research papers will be:

- available free of charge to the entire biomedical community

- peer reviewed and published immediately upon acceptance

- cited in PubMed and archived on PubMed Central

- yours - you keep the copyright

Submit your manuscript here:

http://www.biomedcentral.com/info/publishing_adv.asp
BioMedcentral 